\title{
Grau de risco do pé diabético na atenção primária à saúde
}

Degree of risk of diabetic foot in primary health care

Grado de riesgo de pie diabético en la atención primaria de salud

\section{Patricia Simon da Silva ${ }^{\mathrm{I}}$, Cassandra Severo Amaral Vieira' ${ }^{\mathrm{II}}$, Ludmila Mourão Xavier Gomes ${ }^{\mathrm{III}}$, Thiago Luis de Andrade Barbosa ${ }^{\mathrm{IV}}$}

\begin{abstract}
Resumo: Objetivo: investigar o grau de risco de pé diabético e fatores associados em indivíduos com diabetes mellitus. Método: estudo transversal entre usuários acompanhados em uma unidade básica de saúde. A coleta de dados foi feita com os pacientes que realizaram a consulta de enfermagem por meio de um formulário de avaliação clínica de membros inferiores. Conduziu-se a análise por meio de regressão logística multivariada. Resultados: dos 69 participantes avaliados, 52,8\% apresentaram algum grau de risco. Verificou-se maior chance entre homens, idosos, com relato de dor em repouso nas pernas e pés, histórico de úlcera e/ou amputações, com diagnóstico de enfermagem de "integridade da pele prejudicada no idoso". Conclusão: a maioria dos usuários avaliados não possui risco elevado, porém o enfoque deve ser no autocuidado para a prevenção de tal agravo e a realização do exame clínico dos membros inferiores pelo enfermeiro.
\end{abstract}

Descritores: Diabetes mellitus; Diabetes mellitus tipo 2; Complicações do diabetes; Pé diabético; Processo de enfermagem

\begin{abstract}
Objective: to investigate the risk classification for diabetic foot and associated factors in individuals with diabetes mellitus. Method: a cross-sectional study among users followed up in a basic health unit. Data were collected with patients who underwent the nursing consultation through a clinical evaluation form of lower limbs. The analysis was conducted by means of multivariate logistic regression. Results: total of 69 participants evaluated, $52.8 \%$ presented some degree of risk. There was a higher chance among men, elderly, with reports of pain at rest in the legs and feet, history of ulcer and/or amputations, with nursing diagnosis of "impaired skin integrity in the elderly". Conclusion: most of the users evaluated do not have a high risk, but the focus should be on self-care for the prevention of such injury and the clinical examination of lower limbs by nurse.
\end{abstract}

\footnotetext{
${ }^{\text {I }}$ Enfermeira. Especialista em Saúde da Família. Universidade Federal da Integração Latino Americana (UNILA). Santa Terezinha de Itaipu, Paraná, Brasil. E-mail: patysimon.enf@gmail.com ORCID: https://orcid.org/0000-0002-3998-4962

II Enfermeira. Nefrologista. Universidade Federal da Integração Latino Americana (UNILA). Foz do Iguaçu, Paraná, Brasil. E-mail: cassandra_vieira@hotmail.com ORCID: https://orcid.org/0000-0003-3911-6548

${ }^{\text {III }}$ Enfermeira. Doutora em Ciências da Saúde. Universidade Federal da Integração Latino Americana (UNILA). Foz do Iguaçu, Paraná, Brasil. Email: ludmila.gomes@unila.edu.br ORCID: https://orcid.org/0000-0001-6442-5719

IV Enfermeiro. Doutor em Ciências da Saúde. Universidade Federal da Integração Latino Americana (UNILA). Foz do Iguaçu, Paraná, Brasil. Email: thiago.barbosa@unila.edu.br ORCID: https://orcid.org/0000-0002-6985-9548
} 
Descriptors: Diabetes mellitus; Diabetes mellitus type 2; Diabetes complications; Diabetic foot; Nursing process

Resumen: Objetivo: investigar el grado de riesgo de pie diabético y factores asociados en individuos con diabetes mellitus. Método: estudio transversal entre usuarios seguido en una unidad básica de salud. Se recopilaron datos con pacientes que se sometieron a la consulta de enfermería con una evaluación clínica de las extremidades inferiores. El análisis se realizó mediante regresión logística multivariante. Resultados: del total de 69 participantes, el 52,8\% presentó algún grado de riesgo. Había una mayor probabilidad entre los hombres, ancianos, con informes de dolor en reposo en las piernas y los pies, antecedentes de úlceras y/o amputaciones, con diagnóstico de enfermería de "integridad de la piel deteriorada en los ancianos". Conclusión: la mayoría de los usuarios evaluados no tienen un alto riesgo, pero el enfoque debe estar en el autocuidado para la prevención de tal lesión y el examen clínico de las extremidades inferiores por la enfermera.

Descriptores: Diabetes mellitus; Diabetes mellitus tipo 2; Complicaciones de la diabetes; Pie diabético; Proceso de enfermería

\section{Introdução}

O diabetes mellitus (DM) constitui relevante problema de saúde pública estando associado à qualidade da atenção primária à saúde (APS). Cerca de 415 milhões de pessoas têm DM com prevalência mundial de 8,8\%. Nos países da América Central e do Sul, sua prevalência foi estimada em 26,4 milhões de indivíduos e projetada para 40 milhões em $2030 .{ }^{1}$ O Brasil é o quarto país com o maior número de pessoas com DM no mundo, e estima-se que atualmente 14,3 milhões de brasileiros tenham a doença. Em 2015, ocorreram cinco milhões de mortes por DM em todo mundo, com a proporção de um óbito a cada seis segundos e para o ano de 2040 é previsto que aproximadamente 227 milhões de pessoas desenvolvam a doença. ${ }^{2}$

Em sua fase crônica, o descontrole do perfil glicêmico torna favorável o aparecimento de diversas complicações, dentre as mais graves está o desenvolvimento do pé diabético, condição decorrente da neuropatia que gera perca da sensibilidade, podendo levar ao aparecimento de lesões complexas que, se não tratadas, podem ocasionar amputações de membros inferiores. ${ }^{3}$ Devido ao comprometimento do membro, o pé diabético tem sido uma das principais causas do aumento de internações por condições sensíveis à atenção primária (ICSAP) que atinge cerca de 15,0\% dos indivíduos com DM. ${ }^{4}$ 
As complicações do pé diabético são responsáveis por 40,0\% a 70,0\% do total de amputações de membros inferiores na população geral e $85,0 \%$ das amputações são precedidas de ulcerações. Esse agravo além de aumentar a mortalidade, modifica a qualidade de vida, refletindo em absenteísmo laboral e aposentadorias prematuras. Grande parte desses casos são evitáveis com abordagem educativa ou exame periódico dos pés, pois permitem um tratamento oportuno e evitam o desenvolvimento de complicações. ${ }^{5-6}$

Diante disso, sabe-se que a consulta de enfermagem além de ser considerada uma ferramenta de educação em saúde, possibilita ao profissional desenvolver sua prática de forma autônoma e resolutiva de forma que o enfermeiro desempenhe importante função como agente cuidador e educador. É também de responsabilidade do enfermeiro o exame físico dos pés visando à prevenção do surgimento e agravos relacionados ao pé diabético. ${ }^{7-8}$

Este cenário desperta a necessidade de medidas urgentes para o controle e prevenção do DM, bem como de suas complicações. ${ }^{2}$ Torna-se oportuno a realização de estudos e pesquisas, considerando que, atualmente, há um número expressivo de pessoas que convivem com sequelas graves e expectativa de vida reduzida decorrentes dos danos provocados pela doença. Tendo em vista a gravidade de suas consequências, os dados referentes ao pé diabético são ínfimos no Brasil e, apesar de o exame dos pés ser a forma mais efetiva de rastreamento do pé em risco, ainda não é uma rotina na maioria dos serviços de saúde. ${ }^{9}$ Somado a isso, existe a carência de estudos que tenham abordado a consulta de enfermagem ao paciente com DM com a identificação dos fatores associados do pé diabético. Com base nesses pressupostos, o presente trabalho teve como objetivo investigar o grau de risco de pé diabético e fatores associados em indivíduos com DM.

\section{Método}

Trata-se de estudo transversal realizado com pessoas com DM acompanhadas em uma unidade básica de saúde (UBS) do distrito nordeste de Foz do Iguaçu-Paraná (PR), no período de 
fevereiro a julho de 2019. O município está situado no extremo oeste do PR e faz fronteira com Paraguai e Argentina. Possui população estimada de 258.532 habitantes, destacando-se pelo turismo, comércio e alto fluxo de pessoas em região de tríplice fronteira. ${ }^{10} \mathrm{~A}$ UBS pesquisada possui um total de 3.578 pacientes cadastrados, destes 209 são pessoas com DM.

Foram incluídos na pesquisa os usuários cadastrados e acompanhados pela equipe de saúde da Estratégia de Saúde da Família que possuíam DM há, pelo menos, cinco anos, realizassem consulta de enfermagem no período do estudo e estivessem em tratamento medicamentoso para tal patologia. O convite para participar do estudo ocorreu de quatro formas: contato telefônico; convite por meio do Agente Comunitário de Saúde (ACS); agendamento durante o acolhimento na UBS e abordagem nos grupos educativos de hipertensão e diabetes (HIPERDIA). Foram consideradas perdas aqueles que não foram localizados após três tentativas para agendamento da consulta de enfermagem e os que não compareceram na data e horário agendados para a consulta.

Para realizar a investigação, foi apresentada a proposta de pesquisa e solicitado autorização juntamente com a Secretaria Municipal de Saúde do município e, após aprovação pelo Comitê de Ética em Pesquisa, o projeto foi apresentado a gerente da UBS e à enfermeira responsável pela equipe.

Foi realizado estudo piloto com 10 usuários para verificação e adequação das estratégias de abordagem adotadas e para esclarecimento de possíveis dúvidas sobre o instrumento de coleta de dados. Os indivíduos avaliados nesta etapa foram incluídos na pesquisa, visto que não houve alterações no instrumento e na estratégia de abordagem.

Para a coleta de dados, utilizou-se o formulário denominado "Ficha de avaliação clínica de membros inferiores para prevenção do pé diabético", ${ }^{11}$ o qual foi adaptado à realidade local, composto por quatro fases. A adaptação do instrumento deu-se por razão de alguns dados e/ou testes não serem possíveis de serem coletados ou realizados na UBS estudada pelo fato de não 
5 | Silva PS, Vieira CSA, Gomes LMX, Barbosa TLA

ter os materiais necessários e/ou dados disponíveis, tais como exame de hemoglobina glicada (Hb A1c) >7,0\% nos 3 últimos exames (foi possível coletar dados apenas do último exame, uma vez que a maioria dos pacientes avaliados não possuíam dados de exames anteriores), Retinopatia (pesquisar com fundoscopia), Nefropatia (pesquisar com albuminúria), Avaliação da Sensibilidade Vibratória (com Diapasão de 128 Hz), Avaliação da Motricidade e Reflexo Aquileu.

A primeira fase do instrumento constitui a anamnese que é composta por itens relacionados ao perfil socioeconômico: sexo (masculino/feminino), escolaridade (anos), estado civil (com companheiro/sem companheiro), cor da pele (branca/parda/negra/outra), renda (em salários mínimos - SM). Outra parte dessa fase envolve questões relacionadas à patologia em estudo e fatores de risco para o desenvolvimento do pé diabético, sendo eles: controle glicêmico inadequado no último exame - HbA1c> 7,0\% - (sim/não), idoso (>60 anos), uso de insulina (sim/não), uso de hipoglicemiante oral (sim/não), dislipidemia (hipercolesterolemia, hipertrigliceridemia e HDL-baixo) (sim/não), hipertensão arterial (sim/não), obesidade (IMC >30 Kg/m²) (sim/não) - para tanto foi utilizada uma balança digital com antropômetro adulto da marca Welmy modelo W200A, sedentarismo (sim/não), tabagismo (sim/não), etilismo (sim/não), baixa acuidade visual (sim/não), doenças associadas (sim/não), fatores psicossociais (negação da doença, baixo nível social, morar sozinho) (sim/não) e usar calçados inadequados e/ou andar descalço (sim/não).

A segunda fase do instrumento é constituída por itens relacionados ao exame clínico e classificação de risco do pé diabético que contemplava avaliação geral, como análise antropométrica e avaliações específicas relacionadas ao sistema vascular e neurológico. Também foi avaliada a sensação de pressão com o monofilamento de Semmes-Weinstein de $10 \mathrm{~g}$, sensação térmica utilizando tubos de ensaio com água quente e fria, tátil por meio da utilização de algodão, dolorosa por meio do toque de um palito nos membros inferiores e com base nos resultados obtidos foi classificado o risco de ulceração dos pés. A classificação de risco se deu a partir das diretrizes da Sociedade Brasileira de Diabetes 2013-2014 da seguinte forma: grau de 
Grau de risco do pé diabético na atenção primária à saúde $\mid 6$

risco 0 (paciente sem perca da sensibilidade protetora plantar (PSP), sem doença arterial obstrutiva periférica (DAOP) e sem deformidades), grau de risco 1 (PSP + deformidades), grau de risco 2 (PSP + DAOP) e grau 3 (paciente com úlcera e/ou amputação prévia). ${ }^{12}$

A fase três continha uma lista composta de dez questões com respostas dicotômicas, sim ou não, que avaliavam o autocuidado que o paciente dispensava aos seus pés, tais como a inspeção dos pés, o uso de meias e calçados adequados, a higiene e hidratação dos pés, os cuidados com o corte das unhas e a prevenção de ferimentos. A fase quatro, na qual constavam as orientações a serem seguidas pelos pacientes, foi utilizada em formato de folder entregue aos pacientes no final das consultas, sendo inclusos neste campo os diagnósticos de enfermagem de acordo com a Classificação Internacional das Práticas de Enfermagem em Saúde Coletiva (CIPESC $\left.{ }^{*}\right)$, conforme avaliação e condicionalidades de cada paciente $\cdot{ }^{13} \mathrm{~A}$ coleta de dados ocorreu durante as consultas de enfermagem e duraram, em média, 40 minutos cada, sendo realizadas pela pesquisadora na UBS em dia e horário pactuado com o paciente para a avaliação dos pés, preenchimento da ficha de avaliação dos membros inferiores e posterior classificação de risco. Ao final das consultas, os usuários receberam uma cartilha contendo informações sobre os cuidados necessários para evitar o surgimento de problemas nos membros inferiores.

Os dados foram tabulados no programa Microssoft Excel 2010 e, posteriormente, avaliados utilizando o software Bioestat 5.0 da Universidade Federal do Pará (UFPA). O grau de risco de pé diabético da fase 2 do instrumento foi classificado em ausente (grau 0) e presente (grau 1, 2 e 3), considerada essa a variável dependente do estudo. A associação entre a variável dependente e as independentes foi verificada inicialmente pelo teste de qui-quadrado $\left(\chi^{2}\right)$ e seguida do ajuste de modelo final por meio de regressão logística múltipla, com nível de significância de $5 \%$ realizada com todas variáveis independentes que preencheram critérios de entrada $(\mathrm{p}<0,20)$ em ordem crescente para compor o modelo multivariado final. Dessa forma, permaneceram as variáveis com 
7 | Silva PS, Vieira CSA, Gomes LMX, Barbosa TLA

$\mathrm{p}<0,05$, ou aquelas que alteraram em, no mínimo $10 \%$, o valor de odds ratio (OR). A medida da magnitude de efeito foi verificada pelos valores de OR, e respectivos intervalos de confiança (IC 95\%).

A investigação respeitou as normas éticas de pesquisa que envolve seres humanos da Resolução nº 466/2012 do Conselho Nacional de Saúde/Ministério da Saúde. Para cada participante foi informado os objetivos da pesquisa e que os dados obtidos seriam utilizados exclusivamente para fins científicos, assegurando a confidencialidade, sigilo e o anonimato do participante. A participação se deu mediante assinatura do Termo de Consentimento Livre e Esclarecido. Esta pesquisa foi aprovada pelo Comitê de Ética e Pesquisa do Centro Universitário Dinâmica das Cataratas sob parecer $\mathrm{n}^{\circ} 3.168 .543$ com CAAE $\mathrm{n}^{\circ}$ 07442919.4.0000.8527 na data de 25 de fevereiro de 2019.

\section{Resultados}

Participaram da pesquisa 69 pessoas com DM cadastradas pela equipe de saúde que se enquadraram nos critérios de inclusão. Neste estudo, as perdas foram aquelas que não foram localizados após três tentativas para a realização da consulta de enfermagem (27). Além disso, apenas duas pessoas com DM não aceitaram participar da pesquisa.

Em relação ao perfil dos participantes, constatou-se que a maioria era do sexo feminino (57,9\%; IC $95 \%: 46,3 ; 69,5)$, cor da pele não parda $(59,4 \%$; IC $95 \%: 47,8 ; 71,0)$, viviam com companheiro $\left(62,3 \%\right.$; IC $\left.{ }_{95 \%}: 50,9 ; 73,7\right)$, aposentados $\left(57,9 \%\right.$; IC $\left._{95 \%}: 46,3 ; 69,5\right)$, renda familiar de 02 SM ou mais (50,7\%; IC $95 \%: 38,9 ; 62,5)$, escolaridade maior que 08 anos $(85,5 \%$; IC $95 \%: 77,2 ; 93,8)$, com tempo de duração do DM menor que 05 anos $(72,4 \%$; IC $95 \%: 61,9 ; 82,9)$, não usam insulina $\left(63,7 \%\right.$; IC $95 \%: 52,4 ;_{\text {; }}$ $75,0)$, usam hipoglicemiante oral $\left(97,1 \%\right.$; IC $\left._{95 \%}: 93,1 ; 101,1\right)$ e presença de outras doenças $(85,5 \%$; IC $\left._{95 \%}: 77,2 ; 93,8\right)$.

Em relação ao controle glicêmico $56,5 \%$ (IC $95 \%$ : 44,8; 68,2) possuíam último exame com controle inadequado ( $\mathrm{Hb} \mathrm{A} 1 \mathrm{c}>7,0 \%), 72,4 \%\left(\mathrm{IC}_{95 \%}: 61,9 ; 82,9\right)$ dos entrevistados eram idosos, 
63,7\% (IC $95 \%: 52,4 ; 75,0)$ apresentaram dislipidemia, 79,7\% ( $\left.\mathrm{IC}_{95 \%}: 70,2 ; 89,2\right)$ eram hipertensos, $53,6 \%$ (IC $95 \%: 41,8 ; 65,4)$ estavam com o IMC >30 Kg/m² , um total de $63,7 \%\left(\mathrm{IC}_{95 \%}: 52,4 ; 75,0\right)$ eram sedentários, a maioria declarou não ser tabagista $(94,2 \%$; IC $95 \%: 88,7 ; 99,7)$ nem etilista $(98,5 \%$; IC $\left._{95 \%}: 95,6 ; 101,4\right)$, grande parte dos entrevistados declararam ter baixa acuidade visual $(84,0 \%$; IC $\left._{95 \%}: 75,3 ; 92,7\right)$, a maioria $(75,3 \%$; IC $95 \%: 65,1 ; 85,5)$ não possuíam fatores de risco psicossociais e 72,4\% (IC $95 \%: 61,9 ; 82,9)$ faziam uso de calçados inadequados e/ou andavam descalço.

A Tabela 1 mostra a classificação de risco para desenvolvimento do pé diabético, presente na fase 2 do instrumento. Dos participantes, 47,9\% foram classificados com grau de risco 0 , seguido dos pacientes avaliados com grau de risco $1(39,1 \%)$, grau de risco $3(7,2 \%)$ e $5,8 \%$ classificados com grau de risco 2. Apenas os participantes classificados com grau de risco 3 afirmaram que receberam orientações quanto aos cuidados destinados aos membros inferiores tendo sido avaliados por profissional de saúde previamente.

Tabela 1 - Classificação de risco do pé diabético, Foz do Iguaçu, 2019.

\begin{tabular}{lrr}
\hline \multicolumn{1}{c}{ Variável } & \multicolumn{1}{c}{ n } & \multicolumn{1}{c}{$\%$} \\
\hline Grau 0 & 33 & 47,9 \\
Grau 1 & 27 & 39,1 \\
Grau 2 & 4 & 5,8 \\
Grau 3 & 5 & 7,2 \\
\hline
\end{tabular}

$\mathrm{Na}$ fase 3 do instrumento, foram avaliados aspectos relacionados ao autocuidado destinado aos membros inferiores. Dos participantes avaliados, 53,6\% afirmaram que examinavam os pés diariamente à procura de alterações, $82,6 \%$ referiram andar descalços ou utilizam chinelos com tiras entre os dedos e $75,3 \%$ dos entrevistados relataram que prestavam atenção aos locais por onde andam a fim de evitar ferimentos nos pés.

$\mathrm{Na}$ Tabela 2, encontra-se a análise multivariada que associou estatisticamente na fase 1 do instrumento as variáveis sexo com destaque para os homens e maior chance de pé diabético 
9 | Silva PS, Vieira CSA, Gomes LMX, Barbosa TLA

entre os idosos. Nesta mesma fase os indivíduos com dor em repouso nas pernas e pés e com presença de úlceras e/ou amputações apresentaram maior chance de pé diabético.

Tabela 2 - Análise multivariada das avaliações do pé diabético, Foz do Iguaçu, 2019.

\begin{tabular}{lccc}
\hline \multicolumn{1}{c}{ Variável } & OR & IC $95 \%$ & p \\
\hline Sexo & 1 & & \\
Feminino & 5,02 & 1,$49 ; 16,83$ & 0,009 \\
Masculino & & & \\
Idoso (> 60 anos) & 1 & & \\
Não & 7,70 & 1,$73 ; 34,24$ & 0,007 \\
Sim & & & \\
Dor em repouso nas pernas ou nos pés & 1 & & \\
Não & 8,14 & 1,$21 ; 54,85$ & 0,031 \\
Sim & & & \\
Já teve úlcera ou amputação nos pés & 1 & & \\
Não & 6,78 & 1,$23 ; 37,36$ & 0,028 \\
Sim & & & \\
Integridade da pele prejudicada no idoso & 1 & & \\
Não & 4,70 & 1,$14 ; 19,37$ & 0,032 \\
Sim & & & \\
\hline
\end{tabular}

Na fase 3 do instrumento não houve nenhuma variável com significância estatística para desenvolvimento do pé diabético. $\mathrm{Na}$ fase 4 os indivíduos com diagnóstico de enfermagem de “Integridade da pele prejudicada no idoso" (OR: 4,70; IC ${ }_{95 \%}: 1,14 ; 19,37 ; \mathrm{p}=0.032$ ) mostraram maior chance de pé diabético.

\section{Discussão}

Os achados deste estudo mostraram que a dor em repouso nas pernas e pés e a presença de úlceras e/ou amputações são fatores associados ao desenvolvimento do pé diabético. A maioria dos usuários avaliados não possui risco elevado para o desenvolvimento de tal patologia, porém, o enfoque deve ser no autocuidado para a prevenção de tal agravo. A APS, enquanto porta de entrada, coordenadora e ordenadora da rede de atenção à saúde pode exercer papel 
fundamental para a redução de possíveis complicações, e neste cenário o enfermeiro possui o dever de realizar a avaliação dos pés no intuito de reconhecer os fatores de risco, objetivando diminuir a incidência de pé diabético e amputações. ${ }^{6}$

Neste estudo a maioria das pessoas com DM apresentavam outras comorbidades, como hipertensão arterial e dislipidemia e com controle glicêmico inadequado, o que aponta para a necessidade de um maior acompanhamento da APS como ordenadora do cuidado na rede de atenção à saúde. Pessoas com DM devem ser acompanhados e inseridos em rotinas de cuidados e de ações de educação em saúde, visando o empoderamento para o autocuidado. ${ }^{6}$

Os dados analisados apontaram que $47,9 \%$ dos pacientes avaliados foram classificados com grau de risco 0. Fato semelhante pode ser observado em estudo realizado em São Paulo (SP) em que apesar de o autor classificar o risco de desenvolver o pé diabético de forma distinta em graus 1, 2, 3 e 4, em que o grau 1 corresponde a classificação de risco grau 0 , no presente estudo $66,0 \%$ dos avaliados foram classificados com grau de risco $1 .^{2}$

Apenas aqueles classificados com grau de risco $3(7,2 \%)$ afirmaram que receberam orientações quanto aos cuidados destinados aos membros inferiores e tiveram seus pés avaliados por profissional de saúde, visto que já se encontravam em acompanhamento especializado devido a amputações e/ou úlceras nos membros inferiores. Esse achado aponta para a carência de orientação para o autocuidado do paciente quanto à prevenção do pé diabético, tendo em vista que aqueles pacientes que apresentaram o grau de risco 0,1 e 2 não receberam quaisquer orientações. Investigação conduzida no estado do Ceará, com o intuito de avaliar o conhecimento dos pacientes com DM tipo 2 em relação ao autocuidado com os pés, constatou que 49,8\% possuíam conhecimento inadequado evidenciando que os mesmos seguem orientações de forma fragmentada desconhecendo os riscos associados aos comportamentos adotados. ${ }^{14}$

Outros aspectos evidenciados nesta investigação apontaram que os idosos e homens apresentaram maiores chances de desenvolver pé diabético. Esse fato corrobora com pesquisas 
realizadas em Belo Horizonte (MG) e em um município de grande porte do Sul do Brasil nas quais observaram que a idade avançada tem sido um fator de risco para o aumento da mortalidade em pessoas com DM. No que diz respeito ao autocuidado, os homens apresentaram maiores déficits em comparação às mulheres. ${ }^{15-16}$

Além da maior prevalência de acometimento de doenças crônicas, os idosos apresentam uma condição particular de agravamento das condições de saúde decorrentes de processos fisiopatológicos do envelhecer. ${ }^{17}$ Quando o assunto são os cuidados com a saúde observa-se que os homens são mais suscetíveis ao aparecimento de doenças crônicas não transmissíveis (DCNT) e vivem, em média, sete anos a menos que as mulheres. Esse fato tem se associado, principalmente, à resistência da população masculina em procurar atendimento de saúde preventivamente. ${ }^{18}$

Os indivíduos que apresentaram como principal sintoma dor em repouso em pernas ou pés mostraram ter maior chance de pé diabético. Tal ocorrência representa um problema de saúde por sua característica crônica, podendo gerar incapacidade funcional afetando diretamente a qualidade de vida. Em geral, pessoas com DM que apresentam dor neuropática tem maior comprometimento nas atividades gerais, podendo causar depressão, ansiedade, incluindo distúrbios de sono e repouso. ${ }^{19-20}$ Este sintoma também pode ser devido à aterosclerose que ocorre frequentemente em pessoas com DM por gerar hipóxia tecidual nos membros inferiores. ${ }^{21}$ Estudo de caso realizado em João Pessoa - Paraíba (PB) mostrou que o diagnóstico de enfermagem "Dor intensa em membros inferiores" foi um fenômeno sempre constante, o que aponta que este é um sinal frequente. ${ }^{17}$

O histórico de úlcera e/ou amputações anterior nos pés também se mostrou como fator associado para o desenvolvimento do pé diabético. Estudo longitudinal retrospectivo realizado no Rio Grande do Sul, que avaliou as alterações nos pés de pessoas com DM, mostrou que pacientes com úlcera e amputação apresentaram risco significativo para mortalidade. ${ }^{22}$ Investigação conduzida em Santa Catarina que estimou a carga da doença para as amputações 
de membros inferiores atribuíveis ao DM revelou que os homens sofreram mais amputações de membros inferiores quando comparados às mulheres e que os mesmos perderam mais anos de vida sadia em função da amputação. ${ }^{23}$ Dentre as complicações do DM, a neuropatia diabética destaca-se por ser uma das mais comuns, tornando os pés uma das regiões do corpo mais vulneráveis ao desenvolvimento de úlceras, sendo um fator de risco, considerando que $85,0 \%$ das amputações realizadas nas pessoas com DM são decorrentes de úlcera prévia., ${ }^{2,424}$

Sobre a consulta de enfermagem realizada com as pessoas com DM, destaca-se que esta é uma atividade privativa do enfermeiro que permite ao mesmo exercer sua função de educador em saúde, esclarecendo dúvidas, orientando, promovendo o cuidado e proporcionando ao paciente conhecimento, contribuindo assim na prevenção de agravos oriundos das doenças crônicas. ${ }^{24}$ Dessa forma, a consulta de enfermagem juntamente com a prescrição de cuidados é uma atividade indispensável para o reconhecimento de riscos à saúde, uma vez que oportuniza ao enfermeiro o planejamento de sua assistência. ${ }^{6}$

Dentre os diagnósticos de enfermagem classificados de acordo com a CIPESC ${ }^{\oplus}$ detectouse que aqueles que apresentaram o diagnóstico "Integridade da pele prejudicada no idoso" possuíram chances de 4,70 vezes de desenvolverem o pé diabético do que os pacientes que não apresentaram esse diagnóstico. Esse achado corrobora com pesquisa realizada com idosos com DM em uma UBS de Candido Sales - Bahia que mostrou que o diagnóstico "Risco de integridade da pele prejudicada" foi verificado em todos os participantes do estudo também sendo identificado o diagnóstico efetivo de "Integridade da pele prejudicada” em alguns idosos avaliados. É necessário que o profissional de enfermagem saiba avaliar os membros inferiores dos pacientes e orientá-los quanto aos cuidados necessários, uma vez que a integridade da pele, especialmente dos idosos diabéticos, é prejudicada. ${ }^{25}$

Uma limitação dessa pesquisa se refere ao fato de a metodologia utilizada não permitir averiguar a duração das complicações referente ao pé diabético entre os pesquisados, mas 
apenas identificar a sua presença ou ausência. Nessa perspectiva, este estudo não tem poder de generalização para avaliação da atenção à pessoa com diabetes em outras realidades. Apesar disso, acredita-se que contribui na área de DM e da enfermagem por apresentar os fatores que estão associados ao pé diabético que foram identificados a partir de uma atividade privativa do enfermeiro que é a consulta de enfermagem. Esta investigação aponta para o potencial da ação do enfermeiro na detecção de fatores associados a condições crônicas, como o DM na APS.

\section{Conclusão}

Os homens idosos apresentaram maior risco de desenvolver complicações decorrentes do DM descompensado. O histórico de úlcera e/ou amputações anterior e a presença de dor em repouso em pernas ou pés também mostraram ser fatores de associados ao pé diabético. Detectou-se a ausência da avaliação dos membros inferiores das pessoas com DM na APS, o que pode implicar em futuras complicações do DM por falta de orientação e acompanhamento.

Tal achado aponta para a importância da realização do exame clínico direcionado aos membros inferiores durante as atividades de enfermagem desenvolvidas na APS, pois além de detectar possíveis problemas, possibilita sensibilizar os indivíduos para o autocuidado na prevenção do pé diabético, uma vez que tal problema acarreta na redução da autonomia e autoestima causando impacto na qualidade de vida do indivíduo além de reduzir a capacidade de trabalho e expectativa de vida.

Neste estudo não foi possível compreender quais orientações e ações educação em saúde as pessoas com DM pesquisadas receberam ou participaram. Os níveis de atenção e/ou outros pontos da rede de atenção à saúde que forneceram as orientações educativas também não foram identificados. Dessa forma, recomendam-se investigações futuras que abordem esses aspectos. 


\section{Referências}

1. Ministério da Saúde (BR). Estratégias para o cuidado da pessoa com doença crônica: diabetes mellitus. Brasília (DF): Ministério da Saúde; 2013. (Caderno de Atenção Básica; 36).

2. Lucoveis MLS, Gamba MA, Paula MAB, Morita ABPS. Degree of risk for foot ulcer due to diabetes: nursing assessment. Rev Bras Enferm. 2018;71(6):3041-7. doi: https://doi.org/10.1590/0034-7167-2017-0189

3. Fernandes O, Oliveira P, Pinto CC. The effectiveness of negative pressure therapy for the treatment of diabetic foot wound: an umbrella study. Rev Rol Enferm [Internet]. 2020 [acesso em 2020 mar 07];43(1):405-13. Disponível em: https://comum.rcaap.pt/bitstream/10400.26/31607/1/405-413.pdf

4. Figueiredo EOC, Barros FO, Santos EF, Pimentel TS, Góis CFL, Otero LM. Avaliação do grau de risco para pé diabético em indivíduos com diabetes mellitus tipo 2. Rev Enferm UFPE On Line [Internet]. 2017 [cited 2019 Aug 12];11(Suppl 11):4692-9. Available from: https://periodicos.ufpe.br/revistas/revistaenfermagem/article/view/231211

5. Ministério da Saúde (BR). Manual do pé diabético, estratégias para o cuidado da pessoa com doença crônica. Brasília (DF): Ministério da Saúde; 2016.

6. Nascimento MT, Silva NIO, Brito FCBA, Fontes FLL, Oliveira AF, Oliveira JVA, et al. Fatores de risco associados ao desenvolvimento do pé diabético e ações executadas na Atenção Primária à Saúde para prevenção do agravo. Rev Eletrônica Acervo Saúde. 2019;(33):e1371. doi: https://doi.org/10.25248/reas.e1371.2019

7. Silva KM, Santos SMA. A consulta de enfermagem na estratégia saúde da família: realidade de um distrito sanitário. Rev Enferm UFSM. 2016;6(2):248-58. doi: https://doi.org/10.5902/2179769218079

8. Feitosa TF, Dantas MQS, Silva CB, Pereira A. Monofilament for preventing the diabetic foot: an integrative review of the literature. Online Braz J Nurs [Internet]. 2016 [cited 2019 Aug 03];15(2):291-301. Available from: http://www.objnursing.uff.br/index.php/nursing/article/view/5277/html

9. Gomes LC, Silva Júnior AJ. Fatores favoráveis ao pé diabético em usuários de uma unidade de atenção primária à saúde. Rev Atenção Saúde. 2018;16(57):5-12. doi: https://doi.org/10.13037/ras.vol16n57.4943

10. Instituto Brasileiro de Geografia e Estatística (IBGE). Cidades IBGE. 2019 [acesso em 2019 nov 08]. Disponível em: https://cidades.ibge.gov.br/brasil/pr/foz-do-iguacu/panorama

11. Mello R, Pires M, Kede J. Ficha de avaliação clínica de membros inferiores para prevenção do pé diabético. Rev Pesq Cuid Fundam [Internet]. 2017 [cited 2020 May 25];9(3):899-913. Available from: http://www.seer.unirio.br/index.php/cuidadofundamental/article/view/5468

12. Boulton AJM, Armstrong D, Albert S, Fryberg R, Hellman R, Kirkman M, et al. Comprehensive foot examination and risk assessment. Diabetes Care. 2008;31(8):1679-85. doi: https://doi.org/10.2337/dc08-9021 
13. Albuquerque LM, Cubas MR. CIPESCANDO em Curitiba: construção e implementação da nomenclatura de diagnósticos e intervenções de enfermagem na Rede Básica de Saúde. Curitiba: ABEn; 2005.

14. Oliveira Neto M, Pereira MS, Pinto MAH, Agostinho LM, Reinaldo Júnior FE, Hissa MN. Avaliação do autocuidado para prevenção do pé diabético e exame clínico dos pés em um centro de referência em diabetes mellitus. J Health Biol Sci (Online). 2017;5(3):265-71. doi: http://dx.doi.org/10.12662/23173076jhbs.v5i3.1092.p265-271.2017

15. Cardoso NA, Cisneros LL, Machado CJ, Procópio RJ, Navarro TP. Fatores de risco para mortalidade em pacientes submetidos a amputações maiores por pé diabético infectado. J Vasc Bras (Online). 2017;17(4):296-302. doi: https://doi.org/10.1590/1677-5449.010717

16. Rossaneis MA, Haddad MCFL, Mathias TAF, Marcon SS. Differences in foot self-care and lifestyle between men and women with diabetes mellitus. Rev Lat Am Enferm (Online). 2016; 24:e2761. doi: https://doi.org/10.1590/1518-8345.1203.2761

17. Nogueira LGF, Medeiros ACT, Bittencourt GKGD, Nóbrega MML. Diagnósticos, resultados e intervenções de enfermagem ao idoso diabético: estudo de caso. Online Braz J Nurs [Internet]. 2016 [cited 2019 Aug 08];15(2):302-12. Available from: http://objnursing.uff.br/index.php/nursing/article/download/4964/pdf

18. Lemos APF, Ribeiro C, Fernandes J, Bernardes K, Fernandes RTP. Saúde do homem: os motivos da procura dos homens pelos serviços de saúde. Rev Enferm UFPE On Line [Internet]. 2017 [cited 2019 Oct 13];11(Suppl 11):4645-62. Available from: https://periodicos.ufpe.br/revistas/revistaenfermagem/article/view/231205

19. Cortez J, Reis C, Cardoso Y, Onofre A, Piovezan AP. Prevalência de dor neuropática e fatores associados em portadores de diabetes mellitus tipo 2 atendidos em ambulatório médico. Rev Dor. 2014;15(4):256-9. doi: https://doi.org/10.5935/1806-0013.20140055

20. Girach A, Julian TH, Varrassi G, Paladini A, Vadalouka A, Zis P. Quality of life in painful peripheral neuropathies: a systematic review. Pain Res Manag. 2019; 2019:2091960. doi: 10.1155/2019/2091960

21. Maldonado CM, Silva ACR, Pereira KA, Silva EMA, Oliveira JRC, Faria RS, et al. Associação entre fatores de risco cardiovasculares e a presença de doença arterial coronariana. Arch Med (Manizales). 2019;19(2):247-25. doi: https://doi.org/10.30554/archmed.19.2.3105.2019

22. Scain SF, Franzen E, Hirakata VN. Riscos associados à mortalidade em pacientes atendidos em um programa de prevenção do pé diabético. Rev Gaúcha Enferm. 2018;39:e20170230. doi: https://doi.org/10.1590/1983-1447.2018.20170230

23. Santos KPB, Luz SCT, Mochizuki L, d'Orsi E. Carga da doença para as amputações de membros inferiores atribuíveis ao diabetes mellitus no Estado de Santa Catarina, Brasil, 2008-2013. Cad Saúde Pública. 2018;34(1):e00013116. doi: http://dx.doi.org/10.1590/0102-311x00013116 
24. Oliveira PS, Bezerra EP, Andrade LL, Gomes PLF, Soares MJGO, Costa MML. Atuação dos enfermeiros da estratégia saúde da família na prevenção do pé diabético. Rev Pesq Cuid Fundam [Internet]. $2016 \quad$ [cited 2019 Oct 04];8(3):4841-9. Available from: http://www.seer.unirio.br/index.php/cuidadofundamental/article/view/4398

25. Lacerda NFRS, Lima PV. Diagnósticos de enfermagem identificados em pessoas idosas com diabetes mellitus. ID On Line Rev Psicol [Internet]. 2017 [acesso em 2019 set 23];11(38):431-44. Disponível em: https://idonline.emnuvens.com.br/id/article/view/908/0

Editora Científica: Tânia Solange Bosi de Souza Magnago

Editora Associada: Maria Denise Schimith

\section{Autor correspondente}

Patrícia Simon da Silva

E-mail: patysimon.enf@gmail.com

Endereço: Rua Pedro Machado de Souza, 934, Santa Terezinha de Itaipu, Paraná, Brasil.

CEP: 85875-000

\section{Contribuições de Autoria}

\section{1 - Patrícia Simon da Silva}

Autora principal, realizou seu trabalho de conclusão de residência, o qual deu base para o artigo. Apoiou desde a concepção e planejamento do projeto de pesquisa, coleta de dados a campo, desenvolvimento e análise dos dados assim como redação final do artigo.

\section{2 - Cassandra Severo Amaral Vieira}

Co-orienradora do trabalho, apoiou dede a concepção e planejamento da proposta de pesquisa e coleta de dados.

\section{3 - Ludmila Mourão Xavier Gomes}

Apoiou e participou durante a concepção e planejamento da proposta de pesquisa, análise e interpretação dos dados e redação final do artigo.

\section{4 - Thiago Luis de Andrade Barbosa}

Orientador do trabalho de conclusão, apoiou a concepção e planejamento da proposta de pesquisa, orientou a coleta de dados, realizou análise estatística e revisão de português assim como revisão crítica do artigo.

\section{Como citar este artigo}

Silva PS, Vieira CSA, Gomes LMX, Barbosa TLA. Grau de risco do pé diabético na atenção primária à saúde. Rev. Enferm. UFSM. 2020 [Acesso em: Anos Mês Dia]; vol.10 e78: 1-16. DOI:https://doi.org/10.5902/2179769242614 